\title{
Embolização da artéria gástrica esquerda como alternativa para supressão de apetite e perda de peso em pacientes obesos: uma revisão integrativa
}

\author{
Left gastric artery embolization as an alternative for appetite suppression and weight loss in obese \\ patients: an integrative review \\ Embolización de la arteria gástrica izquierda como alternativa para la supresión del apetito y la \\ pérdida de peso en pacientes obesos: una revisión integrativa
}

Recebido: 31/12/2020 | Revisado: 31/01/2021 | Aceito: 03/02/2021 | Publicado: 09/02/2021

\author{
Mateus Nunes Carvalho \\ ORCID: https://orcid.org/0000-0002-6099-0910 \\ Instituto de Educação Superior do Vale do Parnaíba, Brasil \\ E-mail: mateusnunesc@gmail.com \\ Edvaldo Pereira de Moura Filho \\ ORCID: https://orcid.org/0000-0002-3379-9838 \\ Instituto de Educação Superior do Vale do Parnaíba, Brasil \\ E-mail: edvaldopmourafilho@gmail.com \\ Igor Matheus Oliveira Silva \\ ORCID: https://orcid.org/0000-0002-8627-4015 \\ Instituto de Educação Superior do Vale do Parnaíba, Brasil \\ E-mail: igor_matheussilva@outlook.com \\ Jose Fernandes Neto \\ ORCID: https://orcid.org/0000-0002-4931-0988 \\ Universidade de Fortaleza, Brasil \\ E-mail: jfernandesneto@edu.unifor.br \\ Carlos Augusto Cavalcante de Vasconcelos \\ ORCID: https://orcid.org/0000-0001-8277-7048 \\ Universidade de Fortaleza, Brasil \\ E-mail: ccavalcante20@edu.unifor.br \\ Raissa Pinheiro Nery \\ ORCID: https://orcid.org/0000-0003-3578-3896 \\ Instituto de Educação Superior do Vale do Parnaíba, Brasil \\ E-mail: nery@gmail.com \\ Raissa Martins de Oliveira Nunes \\ ORCID: https://orcid.org/0000-0002-4741-2489 \\ Instituto de Educação Superior do Vale do Parnaíba, Brasil \\ E-mail: raiissamartiins@hotmail.com \\ Ramodnil de Moura Santos \\ ORCID: https://orcid.org/0000-0002-4751-002X \\ Instituto de Educação Superior do Vale do Parnaíba, Brasil \\ E-mail: ramodnilmoura@hotmail.com \\ Luan Kelves Miranda de Souza \\ ORCID: https://orcid.org/0000-0002-8019-4022 \\ Instituto de Educação Superior do Vale do Parnaíba, Brasil \\ E-mail: luankelves11@gmail.com
}

\begin{abstract}
Resumo
A obesidade é caracterizada pelo acúmulo excessivo de gordura corporal a um nível que compromete a saúde dos indivíduos e está associada ao desenvolvimento de doenças crônicas. O tratamento da obesidade consiste na modificação da dieta, instituição de atividade física e farmacoterapia seguida ou não de cirurgia bariátrica. Embora a cirurgia tenha mostrado resultados positivos em relação à perda de peso e reversão de comorbidades, o alto custo, a invasividade, o risco de mortalidade e a restrição da população capaz de realizar o procedimento, fomentam pesquisas por terapias alternativas. Desse modo, existe a embolização de ramos da artéria gástrica esquerda, a fim de promover a supressão do hormônio grelina, um procedimento endovascular para o tratamento da obesidade. O objetivo desta revisão da literatura é analisar os estudos pré-clínicos, clínicos e retrospectivos publicados para avaliar a segurança, eficácia, efeitos colaterais, níveis de grelina e a perda de excesso de peso alcançada após o acompanhamento durante um período. As publicações científicas foram pesquisadas nas bases de dados PubMed, Medline, CINHAL, Scopus, Cochrane e SCIELO de Fevereiro/1999 a Novembro/2020 e 15 estudos foram incluídos. Concluímos ser muito cedo para comparar a eficácia desta técnica com a cirurgia bariátrica, pois os relatórios atuais descrevem apenas a
\end{abstract}


experiência com um número limitado de pacientes com períodos de acompanhamento relativamente curtos. Além disso, a variabilidade entre esses estudos é alta. Preocupações foram levantadas em estudos iniciais sobre o retardo do esvaziamento gástrico que pode ocorrer após esse procedimento, precisando ser avaliado criteriosamente.

Palavras-chave: Cirurgia bariátrica; Embolização terapêutica; Grelina; Obesidade; Perda de peso.

\begin{abstract}
Obesity is characterized by the excessive accumulation of body fat at a level that compromises the health of individuals and is associated with the development of chronic diseases. The treatment of obesity consists of diet modification, institution of physical activity and pharmacotherapy followed or not by bariatric surgery. Although surgery has shown positive results in terms of weight loss and reversal of comorbidities, the high cost, invasiveness, risk of mortality and the restriction of the population capable of performing the procedure, encourage research for alternative therapies. Thus, there is embolization of branches of the left gastric artery, in order to promote the suppression of the hormone ghrelin, an endovascular procedure for the treatment of obesity. The purpose of this literature review is to analyze published pre-clinical, clinical and retrospective studies to assess safety, efficacy, side effects, ghrelin levels and the excess weight loss achieved after follow-up over a period. Scientific publications were searched in the PubMed, Medline, CINHAL, Scopus, Cochrane and SCIELO databases from February/1999 to November/2020 and 15 studies were included. We concluded that it is too early to compare the effectiveness of this technique with bariatric surgery, as current reports describe only the experience with a limited number of patients with relatively short follow-up periods. In addition, the variability between these studies is high. Concerns were raised in initial studies about the delay in gastric emptying that can occur after this procedure, needing to be carefully evaluated.
\end{abstract}

Keywords: Bariatric surgery; Embolization therapeutic; Grelin; Obesity; Weight loss.

\title{
Resumen
}

La obesidad se caracteriza por la acumulación excesiva de grasa corporal a un nivel que compromete la salud de las personas y se asocia al desarrollo de enfermedades crónicas. El tratamiento de la obesidad consiste en la modificación de la dieta, la institución de actividad física y farmacoterapia seguida o no de cirugía bariátrica. Si bien la cirugía ha mostrado resultados positivos en relación a la pérdida de peso y reversión de comorbilidades, el alto costo, la invasividad, el riesgo de mortalidad y la restricción de la población capaz de realizar el procedimiento, incentivan la investigación de terapias alternativas. Por tanto, se produce la embolización de ramas de la arteria gástrica izquierda, para promover la supresión de la hormona grelina, un procedimiento endovascular para el tratamiento de la obesidad. El propósito de esta revisión de la literatura es analizar los estudios preclínicos, clínicos y retrospectivos publicados para evaluar la seguridad, la eficacia, los efectos secundarios, los niveles de grelina y el exceso de pérdida de peso logrado después del seguimiento durante un período. Se realizaron búsquedas de publicaciones científicas en las bases de datos PubMed, Medline, CINHAL, Scopus, Cochrane y SCIELO desde febrero / 1999 hasta noviembre / 2020 y se incluyeron 15 estudios. Concluimos que es demasiado pronto para comparar la efectividad de esta técnica con la cirugía bariátrica, ya que los informes actuales describen solo la experiencia con un número limitado de pacientes con períodos de seguimiento relativamente cortos. Además, la variabilidad entre estos estudios es alta. En los estudios iniciales surgieron inquietudes sobre el retraso en el vaciado gástrico que puede ocurrir después de este procedimiento, lo que debe evaluarse cuidadosamente.

Palabras clave: Cirurgía bariátrica; Embolización terapéutica; Ghrelina; Obesidad; Pérdida de peso.

\section{Introdução}

A obesidade é definida como peso corporal desproporcional à altura com acúmulo excessivo de tecido adiposo, geralmente associado à inflamação sistêmica (Williams et al., 2015). Está associada ao desenvolvimento de doenças crônicas como diabetes mellitus tipo 2, hipertensão, dislipidemia, entre outras (González-Muniesa et al., 2017). Como uma etiologia multifatorial, envolve interações complexas entre genes, hormônios, ambiente e estilo de vida. É uma doença crônica com prevalência crescente. O número de obesos no mundo triplicou desde 1975, totalizando cerca de 650 milhões de pessoas, afetando $13 \%$ da população adulta (Organização Mundial da Saúde [OMS], 2016). Em resposta à obesidade e suas complicações, inúmeras estratégias têm sido preconizadas na tentativa de reduzir sua progressão, a exemplo de intervenções no estilo de vida, tratamento cirúrgico, endoscópico e farmacológico.

As mudanças no estilo de vida (CLS) buscam atuar no controle da massa corporal, considerando o consumo, o gasto energético e a deposição de gordura (González-Muniesa et al., 2017). Estratégias, começando com dieta e prática de atividade física são utilizadas como tratamento de primeira linha para reverter a obesidade, porém, estudos anteriores mostraram que a 
perda de peso varia de 5 a $10 \%$ em pacientes com massa corporal (IMC) entre 25 e $34,9 \mathrm{~kg} / \mathrm{m}^{2}$ (Flegal et al., 2010). No entanto, os pacientes podem retornar ao peso original em até 3 anos após o início da mudança (Christian, Tsai \& Bessesen, 2009).

As opções cirúrgicas têm sido adotadas como abordagem mais invasiva e eficaz, visando emagrecer, melhorar as comorbidades e a qualidade de vida. No entanto, os benefícios da cirurgia são contrabalançados por aspectos negativos precoces, incluindo infecções pós-operatórias, náuseas, vômitos e aspectos tardios, que podem levar à má absorção de vitaminas e minerais (Stefater, Kohli \& Inge, 2013). Terapias endoscópicas como o balão intragástrico e a estimulação gástrica de marca-passo também têm sido utilizadas, entretanto, essas técnicas apresentam diversas complicações e desvantagens.

Os pacientes que não obtiveram êxito no CLS e que não são candidatos à cirurgia bariátrica têm outras opções de tratamento. Existem seis medicamentos aprovados pela Food and Drug Administration (FDA) dos Estados Unidos, que atuam principalmente nas vias do Sistema Nervoso Central (SNC), reduzindo o apetite, aumentando a saciedade ou diminuindo a absorção de gordura. No entanto, a perda de peso alcançada é modesta na faixa de 2,0 a 6,5 kg e a eficácia é limitada, pois a perda de peso permanece apenas enquanto a droga está sendo administrada (Glandt \& Raz, 2011).

Nas últimas décadas, muito se estudou sobre o papel do estômago como órgão endócrino e seu papel no controle homeostático (Wren \& Bloom, 2007). Embora um grande número de hormônios que limitam a ingestão alimentar (anorexígenos) tenha sido descoberto recentemente, o hormônio grelina é o único com efeito oposto (orexígeno) (Vairavamurthy et al., 2017).

A concentração plasmática desse peptídeo é elevada antes das refeições e cai no pós-prandial, sugerindo um papel do apetite e da homeostase energética (Cummings, 2006).

As células produtoras de grelina estão localizadas no fundo gástrico e o suprimento vascular que irriga essa porção do estômago provém predominantemente de ramos que se originam da artéria gástrica esquerda (Gregorczyk \& Agnieszka, 2015; Takiguchi et al., 2011).

Ao embolizar esses ramos, ocorre redução do aporte sanguíneo para a região e, consequentemente, os níveis séricos de grelina diminuem, modificando o balanço homeostático da ingestão energética (Madoff, 2013).

O objetivo desta revisão da literatura é analisar os estudos pré-clínicos, clínicos e retrospectivos publicados para avaliar a segurança, eficácia, efeitos colaterais, níveis de grelina e a perda de peso média alcançada após o acompanhamento durante um período.

\section{Metodologia}

Foi realizada uma revisão sistemática qualitativa, as publicações científicas estão disponíveis na base de dados: PubMed, Medline, CINHAL, Scopus, Cochrane e SCIELO de Fevereiro de 1999 a Novembro de 2020. Os descritores: "artéria gástrica"; "perda de peso"; "obesidade"; "cirurgia bariátrica"; "embolização"; "grelina". As referências dos artigos encontrados foram submetidas a uma revisão manual para verificar a relevância e inclusão dos estudos.

Revisão estruturada de acordo as diretrizes PRISMA (Preferred Reporting Items for Systematic Review and MetaAnalyses). Onde foi realizada as seguintes etapas: 1) Identificação e inclusão dos artigos nas bases de dados selecionadas e revisão das referências, com total de 148 registros identificado por essa triagem inicial; 2) Exclusão de artigos duplicados e análise dos títulos e resumos, realizada por todos os pesquisadores individualmente; 3) Apresentação de apenas 15 de artigos selecionados para compor a pesquisa; 133 de artigos excluídos; 4) Avaliação do texto completo dos artigos para elegibilidade; 5) Apresentação de 133 de artigos excluídos após análise de títulos e resumos, por falta de dados relevantes para pesquisa, como: tipo de estudo, forma e local de intervenção, presença de um grupo controle ou procedimento simulado, tipo de embolização, vasos alvo, medidas de resultado (concentração de grelina, alteração de peso, alteração de gordura subcutânea ou 
medidas de outros parâmetros laboratoriais), complicações pós-procedimento e duração do seguimento; 6) Dos 15 artigos selecionados dividem-se em trabalhos realizados com animais (modelos suínos e modelo canino) e trabalhos realizados com seres humanos. (Moher et al., 2009).

Os critérios de inclusão de estudos na revisão sistemática foram: a) estudos em animais (pré-clínicos) em humanos; b) estudos retrospectivos avaliando a perda de peso em pacientes submetidos à embolização da artéria gástrica esquerda. Como critérios de exclusão definimos: a) artigos publicados em línguas que não inglês, português ou espanhol; b) revisões da literatura, artigos de opinião ou cartas de editores; c) pesquisas que fogem do tema do estudo.

\section{Resultados e Discussão}

A embolização da artéria gástrica esquerda foi introduzida pela primeira vez em 1970 para o tratamento de hemorragia gástrica com risco de vida. Complicações como ulceração isquêmica e infarto, ambos com bom prognóstico, devido à rica circulação colateral do estômago (Prochaska, flye \& Johnsrude, 1973). Com o surgimento de novas técnicas de imagem (micro, seletiva e 3D), dispositivos de acesso vascular (microcátodos, fios-guia, laços) e êmbolos (bobinas, grânulos e microesferas), o espectro desta abordagem foi ampliado e sua aplicação na embolização bariátrica de a artéria gástrica esquerda (Vaidya, Tozer \& Chen, 2008).

A segurança e eficácia desta terapia endovascular para obesidade foram verificadas por vários estudos pré-clínicos (Tabela 1). Arepally et al. (2008) descreveram pela primeira vez o conceito de embolização química da artéria gástrica esquerda para erradicar as células produtoras de grelina no estômago. Uma série de estudos em animais em modelos suínos e cães obesos demonstrou redução pós-embolização dos níveis séricos de grelina e diminuição do ganho de peso em relação ao grupo controle (Arepally et al., 2016).

Tabela 1 - Estudos pré-clínicos de embolização gástrica da artéria gástrica esquerda com o objetivo de reduzir a grelina e a perda de peso.

\begin{tabular}{|c|c|c|c|c|c|c|}
\hline AUTOR & ANO & ESTUDO & GRUPOS & $\begin{array}{c}\text { MUDANÇA NOS } \\
\text { NÍVEIS DE } \\
\text { GRELINA }\end{array}$ & $\begin{array}{c}\text { MUDANÇA DE } \\
\text { PESO }\end{array}$ & EXTRA \\
\hline $\begin{array}{l}\text { Arepally et } \\
\text { al. }\end{array}$ & 2007 & $\begin{array}{l}\text { Estudo } \\
\text { prospectivo, } \\
\text { modelo suíno }\end{array}$ & 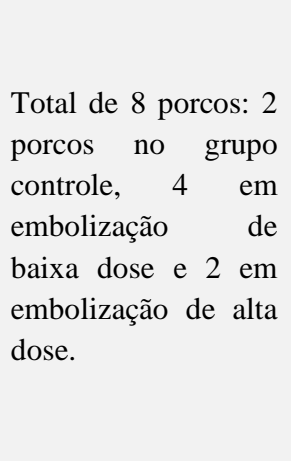 & $\begin{array}{l}\text { Grupo } \quad \text { controle: } \\
\text { nenhuma mudança } \\
\text { significativa, grupo de } \\
\text { dose baixa: aumento } \\
\text { de } 245 \% \pm 34 \% \text {; grupo } \\
\text { de alta dose: aumento } \\
\text { de } 104 \% \pm 23,4 \%\end{array}$ & $\begin{array}{l}\text { Valor médio }( \pm \text { DP) } \\
\text { em } 4 \text { semanas: } \\
\text { grupo } \quad \text { controle, } \\
\text { aumento de } 8,6 \% \pm \\
0,9 \% \text {; embolização } \\
\text { de baixa e alta dose: } \\
\text { aumento de } 1,4 \% \pm \\
10,9 \% ; \quad \text { sem } \\
\text { diferença } \\
\text { estatisticamente } \\
\text { significativa }\end{array}$ & $\begin{array}{l}\mathrm{O} \text { ensaio de } \\
\text { escalonamento de dose } \\
\text { (para cada dose } \\
\text { administrada, } \mathrm{n}=1 \text { ), } \\
\text { produziu resultados } \\
\text { amplamente variáveis }\end{array}$ \\
\hline $\begin{array}{l}\text { Arepally et } \\
\text { al. }\end{array}$ & 2008 & $\begin{array}{l}\text { Estudo } \\
\text { prospectivo, } \\
\text { modelo suíno }\end{array}$ & $\begin{array}{l}\text { Total de } 10 \text { porcos: } 5 \\
\text { no grupo controle e } \\
5 \text { no grupo de } \\
\text { embolização }\end{array}$ & $\begin{array}{l}\text { Grupo } \\
\text { aumento de } 2,6 \% \text { em } 3 \\
\text { semanas e aumento de } \\
18,2 \% \text { em } 4 \text { semanas; } \\
\text { Grupo de embolização, } \\
\text { redução de } 42,5 \% \text { em } \\
3 \text { semanas e redução } \\
\text { de } 12,9 \% \text { em } 4 \\
\text { semanas }\end{array}$ & $\begin{array}{l}\text { Valor médio }( \pm \mathrm{DP}) \\
\text { em } 4 \text { semanas: } \\
\text { grupo } \quad \text { controle, } \\
\text { aumento de } 15,1 \% \pm \\
6 \% \text { Embolização, } \\
\text { aumento de } 7,8 \% \pm \\
5,5 \%\end{array}$ & $\begin{array}{l}\text { Teste de } \\
\text { escalonamento de dose } \\
\text { (para cada dose } \\
\text { administrada, } \mathrm{n}=1 \text { ), } \\
\text { grupo de grande dose }\end{array}$ \\
\hline
\end{tabular}




\begin{tabular}{|c|c|c|c|}
\hline \multirow{6}{*}{$\begin{array}{c}\text { Bawudun et } \\
\text { al. }\end{array}$} & \multirow{6}{*}{2012} & \multirow{6}{*}{$\begin{array}{c}\text { Estudo } \\
\text { prospectivo, } \\
\text { modelo canino }\end{array}$} & $\begin{array}{l}\text { Total de } 15 \text { caninos: } \\
5 \text { no grupo controle, }\end{array}$ \\
\hline & & & $\begin{array}{l}5 \text { no grupo qu } \\
\text { recebeu bleomicina }\end{array}$ \\
\hline & & & $\begin{array}{l}\text { embolização con } \\
\text { óleo estimulado e } \\
\text { no grupo qu }\end{array}$ \\
\hline & & & recebeu partícul \\
\hline & & & PVA \\
\hline & & & embolização \\
\hline
\end{tabular}

Paxton et

al. 2013

Estudo prospectivo, modelo suíno
Total de 12 porcos: 6 no grupo controle e 6 no grupo de embolização

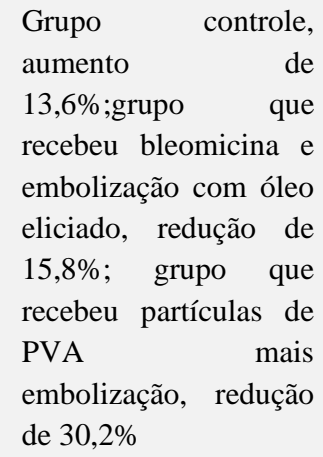

Valor médio ( \pm DP): para o grupo controle, aumento de $328,9 \pm$ $129 \mathrm{pg} / \mathrm{dL}$; para o grupo de embolização, uma diminuição de $537,9 \pm 209,6 \mathrm{pg} / \mathrm{dL}$

\section{Grupo controle: nenhuma mudança significativa. A média ( \pm DP) para o grupo de embolização diminuiu $53,6 \pm 33,43 \mathrm{pg} / \mathrm{dL}$ em 3 semanas e $51,5 \pm$ $15,0 \mathrm{pg} / \mathrm{dL}$ em 5 semanas}

A faixa de partículas de diâmetro de 100$300 \mu \mathrm{m}$ resulta em uma maior redução nas células que expressam grelina do que 300-500 $\mu \mathrm{m}$
Para o grupo de controle, aumento percentual no peso basal;para ambos os grupos de embolização, redução percentual do peso basal (a redução percentual do pico ocorreu na semana 2 ou 3 )

Valor médio ( \pm DP) ao longo de 8 semanas de seguimento: para o grupo controle, aumento de 9,4 \pm $2,8 \mathrm{~kg}$; para o grupo de embolização, aumento de 3,6 \pm $3,8 \mathrm{~kg}$

Valor médio ( \pm DP): para o grupo controle, aumento de $11,5 \pm 6,2 \mathrm{~kg}$; para o grupo de embolização, aumento de 9,4 \pm $0,8 \mathrm{~kg}$

Em porcos tratados com microesferas menores, o ganho de peso médio em 16 semanas foi menor do que em porcos controle
A quantidade de gordura subcutânea também diminuiu nos grupos de embolização vs controle

Os animais controle foram submetidos a um procedimento simulado com $5 \mathrm{~mL}$ de solução salina; A embolização foi realizada com 4-6 mL de microesferas calibradas de $40 \mu \mathrm{m}$ diluídas em solução salina

A embolização foi realizada com partículas não esféricas de PVA 50-150 ou 150-250 $\mu \mathrm{m}$ diluídas em $60 \mathrm{ml}$ de solução salina $50 \%$ e meio de contraste.

Todos os porcos embolizados com as microesferas menores e $60 \%$ dos embolizados com microesferas maiores apresentavam úlceras.

Legenda: PVA = Acetato de polivinila; DP = Desvio padrão. Fonte: Elaborador pelo autor baseado nos estudos relatados na mesma.

O primeiro procedimento realizado em humanos foi apresentado no encontro anual de 2013 do American College of Cardiology, no qual Kipshidze et al. apresentaram estudo prospectivo envolvendo embolização da artéria gástrica esquerda em 5 pacientes com o objetivo de avaliar a segurança e eficácia da técnica com o intuito de reduzir os níveis plasmáticos de grelina e obter perda de peso. Microesferas (Beadblock $®)$ medindo entre 300 e $500 \mu \mathrm{m}$ de diâmetro.

Todos os pacientes relataram diminuição do apetite e IMC foi reduzido em 10\%, 13\%, 16\%, 17\% e 17,2\% após 1, 3, 6, 12 e 24 meses de avaliação. Os níveis plasmáticos de grelina foram significativamente mais baixos no primeiro e terceiro meses (-29\% e -36\%, respectivamente) e diminuíram em até 12 meses (-21\%). Um leve desconforto epigástrico transitório foi descrito d em três dos cinco pacientes (Kipshidze et al., 2015).

Este estudo precedeu um relato de caso publicado por Salsamendi et al. (2015), em que um paciente com $81 \mathrm{~kg}$ e $42 \mathrm{~kg}$ / $\mathrm{m}^{2}$ de IMC de 68 anos com diagnóstico de carcinoma hepatocelular e que necessitou de transplante de fígado por obesidade mórbida não foi candidato à lista de transplante. Durante 7 meses o paciente tentou reduzir o peso com MEV sem sucesso. 
Os médicos responsáveis optaram por realizar a embolização da artéria gástrica esquerda na tentativa de reduzir o peso. Foram utilizadas microesferas (Beadblock (B) medindo entre 500 e $700 \mu \mathrm{m}$ de diâmetro. Sete meses após o procedimento, o paciente apresentava $71,5 \mathrm{~kg}$ e IMC de $36 \mathrm{~kg} / \mathrm{m}^{2}$, sem modificações no estilo de vida, podendo ser colocado na fila de transplante (Salsamendi et al., 2015).

Após esses estudos iniciais, os ensaios clínicos foram realizados. Em 2016, Syed et al. publicaram o Gastric Artery Embolization Trial for the Lessening Appetite Nonurgically (GET LEAN), no qual quatro pacientes com IMC médio de 42,4 $\mathrm{kg} / \mathrm{m}^{2}$ foram submetidos à embolização AGE com microesferas (Beadblock () medindo entre 300 e $500 \mu \mathrm{m}$. Após 6 meses, o IMC médio caiu 8,5\%. Na endoscopia realizada para acompanhamento, $75 \%$ dos pacientes apresentavam ulceração gástrica superficial (Syed et al., 2016).

Já em 2017, Weiss et al. publicaram o "ensaio de embolização arterial bariátrica" (BEAT Obesity) no qual cinco pacientes com IMC superior a $40 \mathrm{~kg} / \mathrm{m}^{2}$ foram submetidos à embolização do fundo gástrico do estômago. Foram utilizadas microesferas Embosphere ( ${ }^{\circledR}$ de 300 a $500 \mu \mathrm{m}$ de diâmetro, com consequente diminuição da grelina em 17,5\% e redução do excesso de peso em $9 \%$ após 3 meses. Os pacientes relataram diminuição do apetite a partir da segunda semana (Weiss et al., 2017).

Ainda no mesmo ano, Bai et al. (2017) avaliaram a segurança do procedimento e os efeitos colaterais em 5 pacientes obesos com IMC médio de $38,1 \mathrm{~kg} / \mathrm{m}^{2}$. Quatro pacientes relataram diminuição do apetite e leve desconforto epigástrico transitório resolvido em 48 horas. Um paciente desenvolveu úlcera, diagnosticada 3 dias após o procedimento por endoscopia, que curou espontaneamente em 30 dias com IBP. Após 9 meses, a perda média de peso foi de 12,64\% (Bai et al., (2017).

O estudo prospectivo mais recente foi publicado em 2018 no qual Pirlet et al. (2018) objetivando avaliar a viabilidade, eficácia e segurança do procedimento. Foram selecionados sete pacientes com obesidade mórbida (IMC médio de $52 \mathrm{~kg} / \mathrm{m}^{2}$ ) que seriam submetidos à cinecoronariografia. Aproveitando o acesso radial, foram injetados cateterismo seletivo da artéria gástrica esquerda (confirmado por angiografia) e partículas de álcool polivinílico (PVA) de 300 a $500 \mu \mathrm{m}$ de diâmetro. Observou-se redução média de $13 \mathrm{~kg}$ após 12 meses do procedimento, correspondendo a redução de 8,13\% na média do IMC (Pirlet et al., 2018).

Além de estudos prospectivos, pesquisas retrospectivas também foram publicadas. Gunn e Oklu (2014), realizaram análise de prontuários de pacientes submetidos à embolização da artéria gástrica esquerda para conter hemorragias do trato gastrointestinal superior. A perda de peso pós-procedimento neste grupo foi comparada com um grupo controle de pacientes que tiveram embolização de outras artérias para sangramento gastrointestinal alto (HDA). Metade do grupo controle tinha história de malignidade e 6 desses pacientes estavam recebendo quimioterapia durante o período do estudo (Anton et al., 2015; Elens et al., 2018; Gunn \& Oklu, 2014; Kim et al., 2017; Takahashi et al., 2019; Zhong, Li \& Ni, 2019). As perdas ponderais do grupo submetido a LGAE foram, em média, de 7,3\% após 1,6 meses o procedimento e 3,5 em 13,6 meses. Essas informações, quando comparado com o grupo controle, evidenciam uma perda de peso mais evidente nos primeiros acompanhamentos.

Anton et al. Realizada uma revisão retrospectiva de pacientes submetidos à embolização arterial por sangramento gastrointestinal superior agudo entre janeiro de 2002 e janeiro de 2014. Pacientes com história documentada de malignidade antes da embolização ou durante o período de acompanhamento foram excluídos para remover o viés de potencial perda de peso do ativo malignidade. Após a exclusão dos pacientes que não atenderam aos critérios de inclusão, o tamanho da amostra foi pequeno, o que limitou o poder do estudo e a significância estatística (Anton et al., 2015).

Em 2018, um estudo retrospectivo de uma única instituição foi realizado por Kim et al. em que os pacientes que foram submetidos à embolização de EGF para tratamento de sangramento gastrointestinal agudo de 2001 a 2016 foram identificados por meio de uma pesquisa de registros médicos de hospitais. Os selecionados tinham entre 33 e 92 anos e não deveriam ter 
diagnóstico de câncer na época do procedimento. A mediana do peso pré-procedimento e do IMC dos pacientes avaliados neste estudo foi de 93,4 kg e 29,9 kg / $\mathrm{m}^{2}$, respectivamente. Após 12 meses foi observada mediana de perda de peso de $16,3 \mathrm{~kg}(\mathrm{p}=$ 0,045) (Kim et al., 2017).

Ainda em novembro do mesmo ano, um estudo belga foi publicado com a finalidade de avaliar a eficácia e segurança da embolização da artéria gástrica esquerda (LGAE) para o tratamento de 17 pacientes com sobrepeso que não eram candidatos à cirurgia bariátrica (IMC médio de $28,9 \mathrm{~kg} / \mathrm{m}^{2}$ ). Apenas um paciente apresentou ulceração gástrica na endoscopia pós-procedimento. Um paciente foi internado na unidade de terapia intensiva por pancreatite e perfuração gástrica. A perda de peso média foi de $8 \mathrm{~kg}$, reduzindo o IMC médio para 25,5 (Elens et al., 2018).

Finalmente em 2019, Takahashi et al. (2019) publicou um estudo retrospectivo com 16 pacientes submetidos ao procedimento entre os anos de 2006 a 2018, com o intuito de determinar em qual parte da composição corporal, como gordura subcutânea ou tecido musculoesquelético, a LGAE apresenta maior efeito no que se refere a diminuição de peso. Para tal, foram selecionados os pacientes com IMC $>25 \mathrm{~kg} / \mathrm{m}^{3}$ submetidos a esse procedimento devido a sangramento gástrico e que possuíam registradas tomografia pré LGAE (1-2 meses) e pós LGAE (1-2 meses) para que estas fossem analisadas e avaliados os índices de gordura corporal total, gordura subcutânea, gordura visceral, índice de gordura intramuscular e índice de muscular esquelético (Takahashi et al., 2019).

O resultado da análise evidenciou que a perda de massa tecidual relacionada ao peso ocorre de forma assimétrica e afeta outras estruturas além do tecido adiposo. Dessa forma, concluiu-se que os pacientes analisados nesse estudo tiveram uma perda considerável de gordura corporal bem como de massa muscular não intencionalmente sugerindo a necessidade de atenção e gerenciamento de peso para que haja um resultado positivo (Takahashi et al., 2019).

Ainda no mesmo ano, Zaitoun et al. (2019) publicou um estudo piloto incluiu 10 pacientes, sendo 7 mulheres e 3 homens, todos obesos e pré-diabéticos com idade média de 37,5 $\pm 8,8$ anos e IMC $>30 \mathrm{~kg} / \mathrm{m}^{2}$, internados para perda de peso entre janeiro de 2017 e junho de 2018. O estudo visava avaliar o impacto do LGAE nesses pacientes, os quais foram analisados HbA1c e o IMC antes do procedimento e 6 meses após, apresentando evidências promissoras quanto a redução clínica do HbA1c e do peso (Gunn \& Weiss, 2019; Zaitoun et al., 2019).

A obesidade é uma doença caracterizada pelo acúmulo excessivo de gordura corporal a um nível que compromete a saúde dos indivíduos, estando associada à comorbidades como diabetes tipo 2, hipercolesterolemia, hipertensão, ataque cardíaco, acidente vascular cerebral, apneia obstrutiva do sono, asma, osteoartrite, flebite, gota, síndrome dos ovários policísticos, infertilidade, depressão e câncer (González-Muniesa et al., 2017).

O diagnóstico da obesidade é baseado no índice de massa corporal (IMC), parâmetro estipulado pela Organização Mundial da Saúde, obtido a partir da relação entre o peso corporal $(\mathrm{kg})$ e a altura $(\mathrm{m})$ dos indivíduos. Por meio desse parâmetro, são considerados obesos os indivíduos com IMC igual ou superior a $30 \mathrm{~kg} / \mathrm{m}^{2}$ (González-Muniesa et al., 2017; World Health Organization [WHO], 2000, p. 253).

$\mathrm{O}$ crescimento geral do índice de massa corporal (IMC) aumentou a morbimortalidade associada à obesidade, o que levou a uma epidemia de saúde pública (Finucane et al., 2011). As principais abordagens terapêuticas para a obesidade incluem mudanças no estilo de vida, intervenção cirúrgica e farmacoterapia.

Mudanças no estilo de vida, incluindo dieta e atividade física, podem ser benéficas para pacientes com sobrepeso (IMC entre 25 e 29,9) e pacientes com obesidade leve (IMC entre 30 e 34,9). Em média, uma perda de peso de 5 a 10\%, entretanto, aqueles que praticam apenas modificação do estilo de vida não apresentam perda de peso sustentada (Aronne et al., 2009; Cawley \& Meyerhoefer, 2012; Jones, Wilson \& Wadden, 2007) 
Medidas mais agressivas, como cirurgia bariátrica (bypass gástrico em Y de Roux, banda gástrica ajustável e gastrectomia vertical), são reservadas para obesos mórbidos (definidos como IMC maior ou igual a 40) ou obesos com IMC acima de 35 e obesidade comorbidade relacionada (Campos et al., 2016).

As complicações pós-operatórias, como vazamento da anastomose, intussuscepção e cálculos biliares, diminuíram com o advento das técnicas laparoscópicas; entretanto, quando ocorrem, podem ser gravemente debilitantes para o paciente, resultando em aumento da morbimortalidade (Baptista \& Wassef, 2013; Reoch, Motillo \& Shimony, 2011).

A abordagem mais bem-sucedida para perda de peso em obesos tem sido a cirurgia bariátrica, no entanto, devido à invasividade, custo e possível morbidade associada a esse procedimento, pesquisas são incentivadas sobre novas alternativas menos invasivas (Bennett, Mehta \& Rhodes, 2007).

Alguns exemplos incluem plicatura gástrica endoscópica, gastroplastia endoscópica de manga, bem como estimuladores elétricos gástricos (Baptista \& Wasseff, 2013). Esses métodos têm se mostrado mais eficazes do que mudanças no estilo de vida, com menor risco de complicações do que a cirurgia bariátrica, e estão sendo avaliados para determinar a segurança em longo prazo mediante a análise de possíveis complicações, vantagens e desvantagens de cada alternativa (Tabela

2) (Kumar \& Thompson, 2011; Verdam et al., 2012).

Tabela 2 - Procedimentos Bariátricos.

\begin{tabular}{|c|c|c|c|c|c|}
\hline PROCEDIMENTOS & TIPO & VANTAGENS & DESVANTAGENS & COMPLICAÇÕES & $(\%$ EWL $)$ \\
\hline Banda gástrica ajustável & Cirurgia & $\begin{array}{l}\text { Reduz a entrada do } \\
\text { estômago } \\
\text { consegue } \\
\text { grande perda de } \\
\text { peso }\end{array}$ & $\begin{array}{l}\text { Os pacientes requerem } \\
\text { acompanhamento } \\
\text { rigoroso e ajustes do } \\
\text { diâmetro interno da } \\
\text { banda com base nos } \\
\text { pesos e sintomas do } \\
\text { paciente. Têm uma taxa } \\
\text { de reoperação de } 5 \% \text { ao } \\
\text { ano }\end{array}$ & $\begin{array}{l}\text { Deslizamento da banda, } \\
\text { prolapso gástrico } \\
\text { cefálico através da } \\
\text { banda, erosão da banda, } \\
\text { dilatação esofágica e } \\
\text { mau funcionamento do } \\
\text { reservatório ou tubo }\end{array}$ & $\begin{array}{l}\text { Atinge uma } \\
\text { média de } \\
44 \% \text { EWL. }\end{array}$ \\
\hline Gastrectomia vertical & Cirurgia & $\begin{array}{l}\text { Está associado a } \\
\text { morbidade mínima, } \\
\text { evita o uso de } \\
\text { material estranho } \\
\text { como banda gástrica } \\
\text { e tem menos } \\
\text { problemas a longo }\end{array}$ & $\begin{array}{l}\text { O procedimento pode } \\
\text { causar vazamentos e } \\
\text { restrições na linha de } \\
\text { grampos }\end{array}$ & $\begin{array}{l}\text { Vazamentos podem não } \\
\text { cicatrizar } \\
\text { espontaneamente, uma } \\
\text { vez que o tubo gástrico } \\
\text { restante opera em alta } \\
\text { pressão }\end{array}$ & $\begin{array}{l}\text { Aos } 5 \text { anos, } \\
\text { o EWL está } \\
\text { entre } 33 \% \text { e } \\
85 \%\end{array}$ \\
\hline
\end{tabular}

\begin{tabular}{|c|c|c|c|c|c|}
\hline Desvio biliopancreático & Cirurgia & $\begin{array}{l}\text { A maioria dos } \\
\text { pacientes mantém a } \\
\text { perda de peso ao } \\
\text { longo de } 21 \text { anos, há } \\
\text { remissão de } 98 \% \text { do } \\
\text { diabetes } 10 \text { anos } \\
\text { após a cirurgia, e a } \\
\text { hipertensão e a } \\
\text { dislipidemia } \\
\text { melhoram }\end{array}$ & 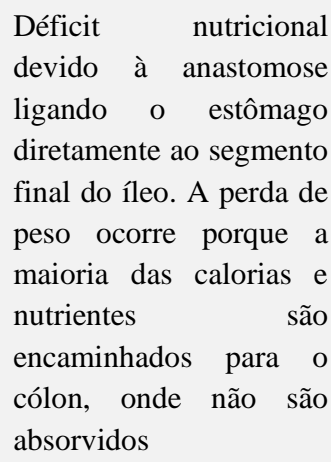 & $\begin{array}{l}\text { Causa diarréia de longo } \\
\text { prazo, anemia, } \\
\text { desnutrição protéica (em } \\
30 \% \text { dos pacientes), } \\
\text { síndrome de dumping, } \\
\text { neuropatia periférica e } \\
\text { osteoporose (má } \\
\text { absorção de cálcio) }\end{array}$ & $\begin{array}{l}\text { Em uma } \\
\text { revisão } \\
\text { histórica de } \\
2.241 \\
\text { pacientes, o } \\
\text { EWL após } \\
1 \text { ano é em } \\
\text { média de } \\
75 \%\end{array}$ \\
\hline
\end{tabular}




\begin{tabular}{|c|c|c|c|c|c|}
\hline $\begin{array}{c}\text { Bypass gástrico em Y de } \\
\text { Roux }\end{array}$ & Cirurgia & $\begin{array}{l}\text { Melhora rápida na } \\
\text { glicoseregulação e } \\
\text { consegue uma } \\
\text { grande perda de } \\
\text { peso }\end{array}$ & 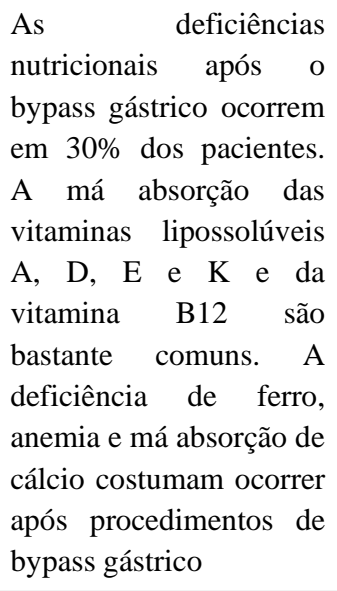 & $\begin{array}{l}\text { Infecção, doença } \\
\text { tromboembólica venosa, } \\
\text { hemorragia, } \\
\text { desenvolvimento de } \\
\text { hérnias ventrais ou intra- } \\
\text { abdominais e obstrução } \\
\text { intestinal. Além disso, } \\
\text { vazamentos } \\
\text { anastomóticos, estenoses } \\
\text { e úlceras podem se } \\
\text { desenvolver }\end{array}$ & $\begin{array}{l}\text { Atinge uma } \\
\text { média de } \\
50 \% \text { EWL }\end{array}$ \\
\hline Balão Intragástrico & Endoscópico & $\begin{array}{l}\text { Preserve a anatomia } \\
\text { do estômago. Os } \\
\text { balões intragástricos } \\
\text { são temporários e o } \\
\text { estímulo que } \\
\text { resultou na perda de } \\
\text { peso é removível. }\end{array}$ & $\begin{array}{l}\text { Entre } 6 \text { e } 15 \% \text { dos } \\
\text { pacientes não toleram o } \\
\text { balão, necessitando de } \\
\text { remoção precoce devido } \\
\text { a náuseas, vômitos e } \\
\text { dores abdominais ou } \\
\text { cólicas persistentes. }\end{array}$ & $\begin{array}{l}\text { O balão pode migrar } \\
\text { causando obstrução do } \\
\text { intestino delgado, } \\
\text { perfuração } \\
\text { (principalmente limitada } \\
\text { a pacientes que já foram } \\
\text { submetidos a cirurgias } \\
\text { no estômago) e piora do } \\
\text { refluxo ácido }\end{array}$ & $\begin{array}{lr}\text { Aos } & 6 \\
\text { meses, } & \\
\text { atinge } & \text { uma } \\
\text { média de } \\
33,9 \% \\
\text { EWL }\end{array}$ \\
\hline Estimulação gástrica & Endoscópico & $\begin{array}{lr}\text { Baixo } & \text { risco } \\
\text { cirúrgico e } & \text { tem } \\
\text { vantagem } & \text { de } \\
\text { permitir ajustes } & \text { no } \\
\text { dispositivo } & \text { de } \\
\text { marcapasso } & \text { sob } \\
\text { demanda } & \end{array}$ & $\begin{array}{l}\text { Todos usam estímulos } \\
\text { que essencialmente não } \\
\text { são percebidos pelos } \\
\text { sujeitos, o que pode } \\
\text { limitar sua eficácia a } \\
\text { longo prazo }\end{array}$ & $\begin{array}{l}\text { Dor no local do } \\
\text { implante, infecção, } \\
\text { perfuração da parede do } \\
\text { estômago, penetração do } \\
\text { chumbo, obstrução } \\
\text { intestinal do chumbo, } \\
\text { problemas com chumbo } \\
\text { / dispositivo, irritação / } \\
\text { inflamação, estimulação } \\
\text { desconfortável } \\
\text { indesejada e danos nos } \\
\text { tecidos, entre outros. }\end{array}$ & $\begin{array}{lr}\text { Em> } & 12 \\
\text { meses, } & \text { em } \\
\text { média, } & \\
\text { houve } & \text { uma } \\
\text { perda de } \\
20 \% \text { do } \\
\text { EWL } \\
\text { nesses } \\
\text { pacientes }\end{array}$ \\
\hline
\end{tabular}

Legenda: EWL = Perda de excesso de peso estimada. Fonte: Elaborado pelo autor baseado em Aldawudi et al. (2020), Allison et al. (2011), Alvarez e Telem (2018), Antel e Hebebrand (2011), Apovian (2016), Arepally et al. (2017) e Piché et al. (2015).

Embora os métodos cirúrgicos tenham um impacto maior na perda de peso percentualmente, observa-se uma gama de efeitos deletérios a curto e longo prazo, visto que desordens funcionais biomecânicas, cicatriciais e nutricional são provocadas por esses procedimentos. Alguns desses percalços também são evidentes nas opções endoscópicas, se sobressaindo pelo nível de gravidade as complicações com repercussões agudas, como perfurações e obstruções, ou que afetam a qualidade de vida como um todo, como mostrado na tabela anterior (Tabela 2).

Também existem medicamentos aprovados para o tratamento da obesidade. A farmacoterapia é atualmente uma terapia adjuvante, não um dos pilares do controle da obesidade. A modulação farmacológica da fome poderia fornecer uma solução para essa epidemia; no entanto, os candidatos a medicamentos atualmente disponíveis mostram uma variação modesta na perda de peso (Tabela 3 ). 
Tabela 3 - Tratamento farmacológico disponível atualmente.

\begin{tabular}{|c|c|c|c|}
\hline MEDICAMENTO & MECANISMO DE AÇÃO & $\begin{array}{c}\text { EFEITOS ADVERSOS } \\
\text { COMUNS }\end{array}$ & PERDA DE PESO \\
\hline Fentermina & $\begin{array}{l}\text { Amina simpaticomimética } \\
\text { que supostamente suprime o } \\
\text { apetite e libera quantidades } \\
\text { insignificantes de dopamina } \\
\text { em comparação com } \\
\text { anfetaminas }\end{array}$ & $\begin{array}{l}\text { Aumento da frequência } \\
\text { cardíaca e / ou pressão arterial, } \\
\text { tontura, boca seca, } \\
\text { constipação, insônia } \\
\text { irritabilidade }\end{array}$ & $\begin{array}{l}\text { Um ensaio clínico de } 2013 \text { relatou uma } \\
\text { perda de peso de } 5.1 \% \text { em } 28 \text { semanas }\end{array}$ \\
\hline Orlistat & $\begin{array}{l}\text { Inibe a atividade das lipases } \\
\text { pancreáticas e gástricas, } \\
\text { diminuindo assim a } \\
\text { absorção de gordura em } \\
30 \%\end{array}$ & 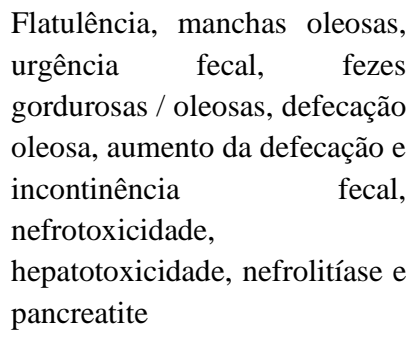 & $\begin{array}{l}\text { Um estudo prospectivo de } 2004 \text { no qual } \\
3.305 \text { pacientes com obesidade foram } \\
\text { aleatoriamente designados para } \\
\text { mudanças no estilo de vida mais } 120 \mathrm{mg} \\
\text { de orlistat ou placebo três vezes ao dia, a } \\
\text { perda de peso média foi estatisticamente } \\
\text { significativa com orlistat }(5,8 \mathrm{~kg} \text { versus } \\
3,0 \mathrm{~kg} \text { com placebo; } \mathrm{P}<0,001)\end{array}$ \\
\hline Fentermina/Topiramato ER & $\begin{array}{l}\text { Simpaticomimético, } \\
\text { aumenta a concentração de } \\
\text { norepinefrina sendo } \\
\text { anorexigênica }\end{array}$ & $\begin{array}{l}\text { Visão turva, parestesia, dor de } \\
\text { cabeça, irritabilidade, tontura, } \\
\text { insônia, depressão e ansiedade }\end{array}$ & $\begin{array}{l}\text { Um ensaio clínico de } 2012 \text { relatou uma } \\
\text { perda de peso corporal de } 9,3 \% \text { no grupo } \\
\text { de } 7,5 \text { / } 46 \mathrm{mg} \text { e uma perda de peso } \\
\text { corporal de } 10,5 \% \text { no grupo de } 15 / 92 \mathrm{mg} \\
\text { em comparação com o grupo de placebo } \\
(-1,8 \% \text {; }<<0,0001)\end{array}$ \\
\hline Lorcaserin & $\begin{array}{l}\text { Um agonista 5HT2C que } \\
\text { atua nos neurônios POMC } \\
\text { anorexígenos no hipotálamo }\end{array}$ & $\begin{array}{l}\text { Náusea, boca seca, dor de } \\
\text { cabeça, tontura, fadiga e } \\
\text { constipação }\end{array}$ & $\begin{array}{l}\text { Um ensaio clínico randomizado } \\
\text { controlado por placebo de } 2012 \text { relatou } \\
45,0 \% \text { de perda de peso corporal em } \\
\text { comparação com o grupo placebo (- } \\
1,5 \% \text {; }<0,001)\end{array}$ \\
\hline Naltrexona S/ Bupropiona SR & $\begin{array}{l}\text { A naltrexona bloqueia a } \\
\text { autoinibição de POMC } \\
\text { mediada por receptor de } \\
\text { opioide e a bupropiona inibe } \\
\text { seletivamente a recaptação } \\
\text { de dopamina } \\
\text { noradrenalina } \\
\text { antidepressivo }\end{array}$ & $\begin{array}{l}\text { Náusea, constipação, dores de } \\
\text { cabeça, vômitos, tontura e boca } \\
\text { seca }\end{array}$ & $\begin{array}{l}\text { Um ensaio clínico randomizado de } 2013 \\
\text { relatou uma perda de peso corporal de } \\
6,4 \% \text { no grupo de } 32 / 360 \mathrm{mg} \mathrm{em} \\
\text { comparação com o grupo de placebo (- } \\
1,2 \% \text {; }<0,001)\end{array}$ \\
\hline Liraglutida & $\begin{array}{l}\text { Um agonista do receptor } \\
\text { GLP1 de um hormônio } \\
\text { derivado da incretina que } \\
\text { atua através das vias do } \\
\text { receptor periférico e central } \\
\text { afetando a homeostase da } \\
\text { glicose, a ingestão de } \\
\text { alimentos e a saciedade. }\end{array}$ & $\begin{array}{l}\text { Náusea, diarreia, constipação, } \\
\text { vômito, dispepsia e dor } \\
\text { abdominal. }\end{array}$ & $\begin{array}{l}\text { Em um ensaio clínico randomizado } \\
\text { controlado de } 2015 \text {, um total de } 63,2 \% \\
\text { dos pacientes no grupo liraglutida em } \\
\text { comparação com } 27,1 \% \text { no grupo } \\
\text { placebo perderam pelo menos } 5 \% \text { do } \\
\text { peso corporal (P <0,001) e } 33,1 \% \text { e } \\
10,6 \% \text {, respectivamente, perderam mais } \\
\text { de } 10 \% \text { do peso corporal }(\mathrm{P}<0,001 \text { ). }\end{array}$ \\
\hline
\end{tabular}

Legenda: GLP1 = peptídeo 1 semelhante ao glucagon; 5HT2C = receptor 5-hidroxitriptamina 2C; POMC = Pró-opiomelanocortina. Fonte: Elaborado pelo autor baseado em Allison et al. (2011), Antel e Bebebrand (2011), Bawudun et al. (2012), Buchwald et al. (2004), Fu et al. (2018), Gadde et al. (2011), Hendricks et al. (2013), Kim et al. (2018), O’Neil et al. (2012); Paxton et al. (2013), Piché et al. (2015), PiSunyer et al. (2015), Weir (2011) e Weiss e Kathait (2017).

A adesão a longo prazo à terapia medicamentosa continua sendo um desafio constante devido ao alto custo e aos consideráveis efeitos adversos associados a essa terapia, além de impactarem de forma mais modesta a perda de peso desses pacientes, como evidencia a Tabela 3 (Coutinho \& Glancey, 2013; Maahs et al., 2006). 
A embolização de ramos da artéria gástrica esquerda é uma alternativa para o tratamento da obesidade, por ser um procedimento minimamente invasivo baseado nos efeitos hormonais da cirurgia bariátrica (Weiss et al., 2015).

A justificativa para a embolização bariátrica como alvo no tratamento da obesidade decorre do papel do Ghrel em um peptídeo de 28 aminoácidos, secretado principalmente pelo fundo do estômago (90\%) (Wren \& Bloom, 2007). Atua no estímulo do apetite e induz a fome, aumentando a ingestão alimentar e resulta em ganho de peso (Cummings \& Overduin, 2007; Kojima et al., 1999).

F igura 1 - Papel da regulação da homeostase energética.

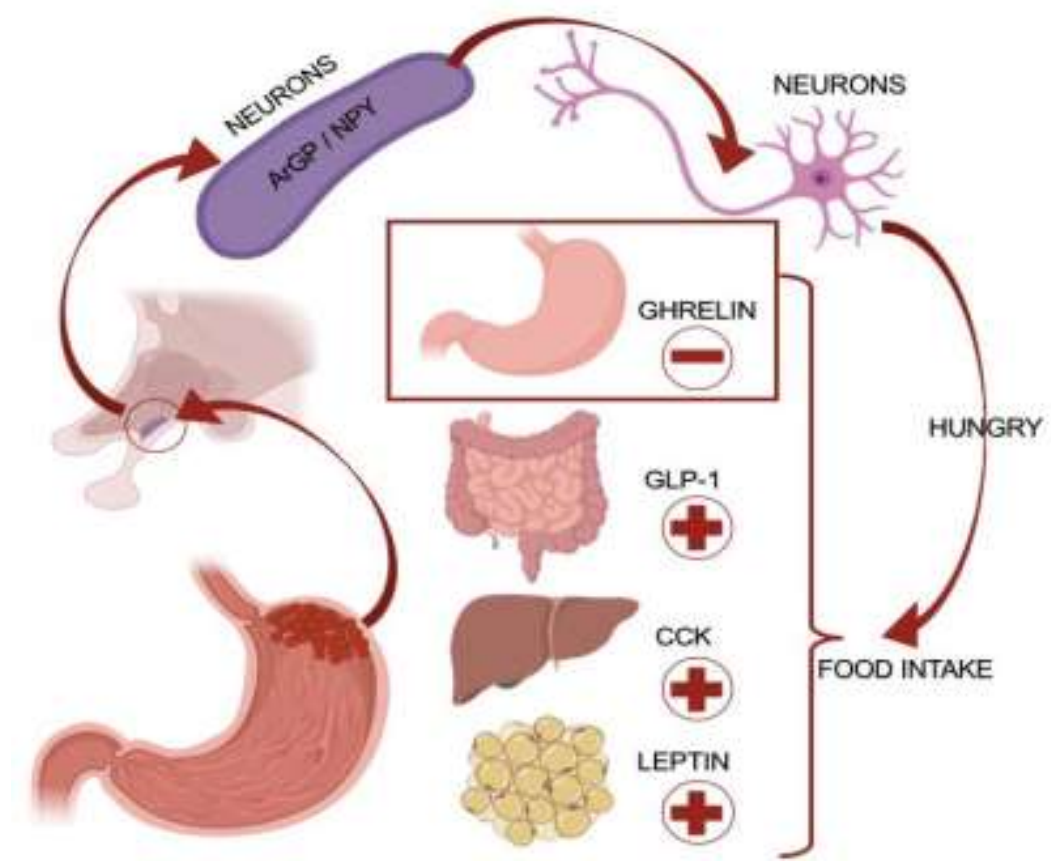

Texto: Com efeito intestinal, as células de fundo produzem grelina, que atuará sobre o neuropeptídeo Y (NPY) e o peptídeo cutia (AGRP) presentes nos núcleos do hipotálamo. A liberação de gordura é uma liberação de gordura decorrente da dilatação do estômago e estimula a liberação de leptina pelo tecido adiposo, GLP-1 pelo intestino e CCK pelo fígado, responsável pela saciedade. Os níveis de grelina são tributados pela fome. Fonte: Cummings (2006).

Fisiologicamente, os níveis plasmáticos de grelina aumentam significativamente antes das refeições (induzindo fome) e tem seu declínio no período após a alimentação (como parte da resposta de saciedade), o que a caracteriza como um hormônio orexígeno (Figura 1) (Cummings et al., 2001; Wren et al., 2001).

No entanto, os níveis de grelina não diminuem quando o paciente obeso se alimenta, o que pode contribuir para a alimentação em excesso (English et al., 2002). Além disso, esse peptídeo pode ser parcialmente responsável pela perda de peso após a cirurgia bariátrica (Cummings et al., 2002).

Ao criar isquemia principalmente no fundo gástrico, a embolização bariátrica visa atingir as células produtoras de grelina a fim de reduzir seus níveis plasmáticos e, assim, reduzir a fome, contribuindo para a perda de peso em pacientes obesos (Weiss \& Kathait, 2017).

Outros impactos positivos ainda carecem de mais evidências, como repercussão positiva no perfil lipídico, glicemia média e síndrome metabólica dos pacientes submetidos a LGAE, contudo os ensaios parecem evidenciar que há efeito benéfico do procedimento para pacientes com essas comorbidades, com boa repercussão clínica nas avaliações posteriores (Aldawudi et al., 2020). 
Esse procedimento pode ser considerado uma ponte para outros procedimentos, conforme sugerido em relato de caso publicado recentemente que descreve a embolização da artéria gástrica esquerda em paciente obeso com carcinoma hepatocelular aguardando cirurgia. A questão de saber se esse procedimento pode ser usado como uma ponte para outros procedimentos bariátricos está longe de ser resolvida.

Ainda, faz-se necessário esclarecer qual o perfil de candidato ideal para LGAE eletiva, visto que os ensaios disponíveis apresentam perfis diferentes de IMC (inferior a $40 \mathrm{~kg} / \mathrm{m}^{2}$ e $30 \mathrm{~kg} / \mathrm{m}^{2}$ em GET LEAN e BEAT Obesity, respectivamente), embora ambos tenham apresentado faixa de perda de peso semelhante (Zhong, Li \& Ni, 2019).

Pode ser muito cedo para comparar a eficácia desta técnica com a cirurgia bariátrica, uma vez que os relatórios atuais descrevem apenas a experiência com um número limitado de pacientes com períodos de acompanhamento relativamente curtos. Além disso, a variabilidade entre esses estudos é alta (Tabela 4).

Tabela 4 - Estudos prospectivos e retrospectivos de embolização da artéria gástrica esquerda em humanos.

\begin{tabular}{|c|c|c|c|c|c|c|c|c|c|c|c|}
\hline $\begin{array}{c}\text { AUTOR / } \\
\text { ANO }\end{array}$ & (n) & $\begin{array}{c}\text { MAS } \\
\text { C. }\end{array}$ & FEM. & ESTUDO & $\begin{array}{c}\text { AGENTE } \\
\text { EMBÓLI } \\
\text { CO }\end{array}$ & $\begin{array}{c}\text { TAMA } \\
\text { NHO } \\
\text { DO } \\
\text { AGENT } \\
\text { E } \\
\text { EMBO } \\
\text { LICO }\end{array}$ & $\begin{array}{c}\text { ACOM } \\
\text { PANHA } \\
\text { MENT } \\
\text { O }\end{array}$ & $\begin{array}{c}\text { OBJET } \\
\text { IVO } \\
\text { PRIMÁ } \\
\text { RIO }\end{array}$ & $\begin{array}{c}\text { RESUL } \\
\text { TADOS }\end{array}$ & $\begin{array}{c}\text { EVENTO } \\
\text { S } \\
\text { ADVERS } \\
\text { OS }\end{array}$ & IMC \\
\hline $\begin{array}{c}\text { Gunn \& } \\
\text { Oklu } \\
(2014)\end{array}$ & 19 & 12 & 7 & $\begin{array}{c}\text { Retrospectiv } \\
\text { o }\end{array}$ & $\begin{array}{l}\text { Bobinas, } \\
\text { Gelfoam, } \\
\text { PVA }\end{array}$ & $\begin{array}{l}300- \\
500, \\
500- \\
710, \\
710- \\
1000\end{array}$ & $\begin{array}{c}14 \\
\text { meses }\end{array}$ & $\begin{array}{c}\text { Perda de } \\
\text { peso }\end{array}$ & $\begin{array}{l}\text { Redução } \\
\text { do IMC } \\
\text { em } 7,3 \% \\
\text { nos } \\
\text { primeiro } \\
\text { s } 45 \text { dias } \\
\text { e } \\
\text { redução } \\
\text { de } 3,5 \% \\
\text { em } 13 \\
\text { meses. }\end{array}$ & $\begin{array}{l}\text { Desconhe } \\
\text { cido }\end{array}$ & 30,3 \\
\hline $\begin{array}{l}\text { Anton et } \\
\text { al. (2015) }\end{array}$ & 10 & 7 & 3 & $\begin{array}{c}\text { Retrospectiv } \\
0\end{array}$ & $\begin{array}{l}\text { Bobinas, } \\
\text { Gelfoam }\end{array}$ & $\mathrm{N} / \mathrm{D}$ & $\begin{array}{c}12 \\
\text { meses }\end{array}$ & $\begin{array}{l}\text { Perda de } \\
\text { peso }\end{array}$ & 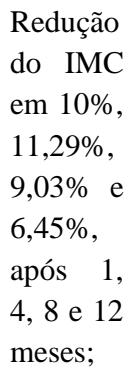 & $\begin{array}{l}\text { Desconhe } \\
\text { cido }\end{array}$ & 31 \\
\hline $\begin{array}{l}\text { Kipshidze } \\
\text { et al. } \\
\text { (2015) }\end{array}$ & 5 & 4 & 1 & Prospectivo & $\begin{array}{l}\text { Bead } \\
\text { block® }\end{array}$ & $300-500$ & $\begin{array}{c}24 \\
\text { meses }\end{array}$ & $\begin{array}{l}\text { Seguran } \\
\text { ça e } \\
\text { Eficácia }\end{array}$ & 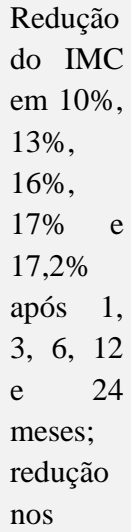 & $\begin{array}{l}\text { Desconfor } \\
\text { to } \\
\text { epigástric } \\
\text { o } \\
\text { transitório } \\
\text { leve }\end{array}$ & 42,2 \\
\hline
\end{tabular}




\begin{tabular}{|c|c|c|c|c|c|c|c|c|c|c|c|}
\hline & & & & & & & & & $\begin{array}{l}\text { níveis } \\
\text { plasmáti } \\
\text { cos de } \\
\text { grelina }\end{array}$ & & \\
\hline $\begin{array}{l}\text { Salsamend } \\
\text { i et al. } \\
\text { (2015) }\end{array}$ & 1 & 0 & 1 & Prospectivo & $\begin{array}{l}\text { Microesfer } \\
\text { as } \\
\text { Embosphe } \\
\text { re }{ }^{\circ}\end{array}$ & $500-700$ & 7 meses & $\begin{array}{l}\text { Perda de } \\
\text { peso }\end{array}$ & $\begin{array}{l}\text { Redução } \\
\text { do IMC } \\
\text { em } \\
14,3 \% \text { e } \\
\text { redução } \\
\text { da } \\
\text { esteatose } \\
\text { hepática }\end{array}$ & $\begin{array}{l}\text { Sem } \\
\text { complicaç } \\
\text { ões } \\
\text { relatadas }\end{array}$ & 42 \\
\hline $\begin{array}{l}\text { Syed et al. } \\
\text { (2016) }\end{array}$ & 4 & 1 & 3 & Prospectivo & $\begin{array}{l}\text { Bead } \\
\text { block } ®\end{array}$ & $300-500$ & 6 meses & $\begin{array}{l}\text { Seguran } \\
\text { ça para o } \\
\text { procedi } \\
\text { mento }\end{array}$ & $\begin{array}{l}\text { Redução } \\
\text { do IMC } \\
\text { em } \\
8,5 \% \text {, } \\
\text { melhora } \\
\text { na } \\
\text { hemoglo } \\
\text { bina } \\
\text { glicada } \\
\text { de } 7,4 \% \\
\text { para } \\
6,3 \% \text { em } \\
6 \text { meses }\end{array}$ & $\begin{array}{l}\text { Náusea } \\
\text { com } \\
\text { vômitos } \\
\text { ocasionais } \\
\text { desconfort } \\
\text { o leve } \\
\text { epigástric } \\
\text { o } \\
\text { transitório }\end{array}$ & 42,4 \\
\hline $\begin{array}{c}\text { Bai et al. } \\
\text { (2017) }\end{array}$ & 5 & 3 & 2 & Prospectivo & PVA & $500-710$ & 9 meses & $\begin{array}{l}\text { Seguran } \\
\text { ça para o } \\
\text { procedi } \\
\text { mento }\end{array}$ & 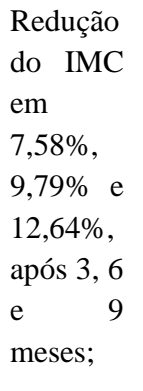 & $\begin{array}{l}\text { Ulceração } \\
\text { de } \\
\text { superfície } \\
\text { com } \\
\text { hematoma } \\
\text { no local } \\
\text { da punção }\end{array}$ & 38,1 \\
\hline $\begin{array}{l}\text { Weiss et } \\
\text { al. (2017) }\end{array}$ & 5 & 1 & 4 & Prospectivo & $\begin{array}{l}\text { Microesfer } \\
\text { as } \\
\text { Embosphe } \\
\text { re } ₫\end{array}$ & $300-500$ & 3 meses & $\begin{array}{l}\text { Seguran } \\
\text { ça para o } \\
\text { procedi } \\
\text { mento }\end{array}$ & $\begin{array}{l}\text { A } \\
\text { mudança } \\
\text { média } \\
\text { na } \\
\text { grelina } \\
\text { sérica } \\
\text { foi de } \\
8,7 \% \quad \pm \\
34,7 \text { e - } \\
17,5 \% \pm \\
29 \text { em } 1 \\
\text { mês e } 3 \\
\text { meses, } \\
\text { respectiv } \\
\text { amente }\end{array}$ & $\begin{array}{l}\text { Pancreatit } \\
\text { e } \\
\text { transitória, } \\
\text { ulceração } \\
\text { superficial } \\
\text { assintomát } \\
\text { ica }\end{array}$ & 43,8 \\
\hline $\begin{array}{l}\text { Elens et al. } \\
\text { (2018) }\end{array}$ & 16 & 2 & 14 & $\begin{array}{c}\text { Retrospectiv } \\
\text { o }\end{array}$ & $\begin{array}{l}\text { Microesfer } \\
\text { as } \\
\text { Embosphe } \\
\text { re } ®\end{array}$ & $\begin{array}{l}300- \\
500, \\
500-700\end{array}$ & $\begin{array}{c}12 \\
\text { meses }\end{array}$ & $\begin{array}{l}\text { Seguran } \\
\text { ça e } \\
\text { Eficácia }\end{array}$ & $\begin{array}{l}\text { Redução } \\
\text { do IMC } \\
\text { em } 10 \% \\
\text { após } 6\end{array}$ & $\begin{array}{l}6 \% \\
\text { tiveram } \\
\text { uma } \\
\text { ulceração } \\
\text { gástrica } \\
\text { superficial } \\
\text { observada }\end{array}$ & 28,9 \\
\hline
\end{tabular}




\begin{tabular}{|c|c|c|c|c|c|c|c|c|c|c|c|}
\hline & & & & & & & & & meses & $\begin{array}{l}\text { na } \\
\text { endoscopi } \\
\text { a após a } \\
\text { intervençã } \\
\text { o }\end{array}$ & \\
\hline $\begin{array}{l}\text { Kim et al. } \\
\text { (2018) }\end{array}$ & 12 & 9 & 0 & $\begin{array}{c}\text { Retrospectiv } \\
\text { o }\end{array}$ & $\begin{array}{l}\text { Bobinas, } \\
\text { Gelfoam, } \\
\text { PVA }\end{array}$ & $\begin{array}{l}300-500 \\
500-700\end{array}$ & $\begin{array}{c}12 \\
\text { meses }\end{array}$ & $\begin{array}{l}\text { Perda de } \\
\text { peso }\end{array}$ & $\begin{array}{l}\text { Redução } \\
\text { do IMC } \\
\text { em } \\
9,36 \% \\
\text { em um } \\
\text { tempo } \\
\text { médio } \\
\text { de } 12 \\
\text { meses }\end{array}$ & $\begin{array}{l}\text { ulceração } \\
\text { superficial }\end{array}$ & 29,9 \\
\hline $\begin{array}{l}\text { Pirlet et al. } \\
\text { (2018) }\end{array}$ & 7 & 7 & 0 & Prospectivo & PVA & $\begin{array}{l}300-500 \\
100-300\end{array}$ & $\begin{array}{c}12 \\
\text { meses }\end{array}$ & $\begin{array}{l}\text { Perda de } \\
\text { peso }\end{array}$ & $\begin{array}{l}\text { Redução } \\
\text { do IMC } \\
\text { em } \\
4,38 \%, \\
3,75 \%, \\
8,18 \% \\
\text { em 2, } 6 \\
\text { e } 12 \\
\text { meses, } \\
\text { respectiv } \\
\text { amente }\end{array}$ & $\begin{array}{l}\text { dor } \\
\text { abdominal } \\
\text { leve } \\
\text { transitória }\end{array}$ & 52 \\
\hline $\begin{array}{l}\text { Zaitoun et } \\
\text { al. (2019) }\end{array}$ & 10 & 3 & 7 & Prospectivo & $\begin{array}{l}\text { Microesfer } \\
\text { as } \\
\text { Embosphe } \\
\text { re } \AA\end{array}$ & $300-500$ & 6 meses & $\begin{array}{l}\text { Perda de } \\
\text { peso }\end{array}$ & $\begin{array}{l}\text { Redução } \\
\text { do IMC } \\
\text { e HbA1c } \\
\text { em } 8,8 \% \\
\text { e } 8,9 \% \text {, } \\
\text { respectiv } \\
\text { amente, } \\
\text { em um } \\
\text { tempo } \\
\text { médio } \\
\text { de } 6 \\
\text { meses }\end{array}$ & $\begin{array}{l}\text { Apenas } \\
\text { dor } \\
\text { epigástrica } \\
\text { leve } \\
\text { ocorreu } \\
\text { em } 7 \\
\text { pacientes } \\
(70 \%) \text { e } \\
\text { desaparec } \\
\text { eu em } 24 \\
\text { horas. }\end{array}$ & 34,1 \\
\hline
\end{tabular}

Fonte: Autores, baseado nos estudos relatados na mesma.

Conforme o exposto (Tabela 4), os efeitos adversos secundários ao procedimento têm um impacto diminuto na situação de saúde do paciente como um todo, quando posto em paralelo a outros métodos mais invasivos. Tal evidência, somada à eficácia demonstrada pelos estudos em questão, podem elevar a embolização da artéria gástrica a opção viável a ser analisada.

Em comparação, a cirurgia bariátrica possui uma riqueza de dados que confirma a perda sustentada ( $\geq 6$ anos) entre $22 \%$ e $55 \%$ do peso corporal pré-intervenção, dependendo do tipo de cirurgia bariátrica realizada (Piché et al., 2015). Da mesma forma, no que diz respeito às complicações, o perfil de segurança da embolização bariátrica não pode ser inferido da experiência limitada disponível com essa técnica.

\section{Conclusão}

A utilização da técnica intervencionista de embolização da artéria gástrica esquerda já é amplamente utilizada para o tratamento do sangramento gastrointestinal. No entanto, esse procedimento em um cenário eletivo como tratamento da 
obesidade ainda carece de pesquisas que comprovem eficácia e segurança em longo prazo. Vários obstáculos permanecem no caminho para iniciar esta terapia para uso principal na perda de peso.

Ainda não há consenso sobre qual material de embolização seria ideal se pequenas partículas ou agentes esclerosantes líquidos proporcionam o melhor perfil para obter oclusão vascular duradoura e penetrar em capilares menores, evitando embolização não alvo e lesão tecidual associada.

Há, também, a falta de consenso quanto a técnica e abordagem mais eficazes quanto a LGAE no tratamento da obesidade, embora geralmente seja feita através de embolização transarterial guiada por fluoroscopia e indica-se usualmente a abordagem transradial em detrimento da abordagem transfemoral por essa última ser potencialmente mais arriscada. Além disso, surgiram preocupações nos primeiros estudos sobre o retardo do esvaziamento gástrico que pode ocorrer após este procedimento. Isso precisaria ser avaliado mais formalmente. Uma vez que um perfil de segurança aceitável seja estabelecido, ensaios prospectivos, multicêntricos e randomizados podem ser realizados.

Ademais, os riscos que envolvem sarcopenia e fragilidade muscular devem ser considerados e analisados para que possa ser feito manejo multiprofissional adequado dessa repercussão possivelmente deletéria após o procedimento.

Portanto, conclui-se que os esclarecimentos vindouros concernentes a técnica e materiais mais adequados a serem utilizados, bem como os riscos e complicações a curto e longo prazo relacionados ao procedimento, além das repercussões na condição de saúde global do paciente, tanto no que se refere à eficácia quanto efeitos colaterais, são informações fundamentais para melhor desenvolvimento dessa possibilidade terapêutica.

\section{Referências}

Aldawudi, I., Katwal, P. C., Jirjees, S., Htun, Z. M., \& Khan, S. (2020). Future of Bariatric Embolization: A Review of Up-to-date Clinical Trials. Cureus, 12(5), e7958. https://doi.org/10.7759/cureus.7958

Allison, D. B., Gadde, K. M., Garvey, W. T., Peterson, C. A., Schwiers, M. L., Najarian, T., Tam, P. Y., Troupin, B., \& Day, W. W. (2012). Controlled-release phentermine/topiramate in severely obese adults: a randomized controlled trial (EQUIP). Obesity (Silver Spring, Md.), 20(2), 330-342. https://doi.org/10.1038/oby.2011.330

Alvarez, R., \& Telem, D. A. (2018) Comparative Surgical Outcomes in Bariatric Surgery. In The SAGES Manual of Bariatric Surgery (pp.339-361). EUA: Springer International Publishing. http://dx.doi.org/10.1007/978-3-319-71282-6_29.

Antel, J., \& Hebebrand, J. (2012). Weight-reducing side effects of the antiepileptic agents topiramate and zonisamide. Handbook of experimental pharmacology, (209), 433-466. https://doi.org/10.1007/978-3-642-24716-3_20

Anton, K., Rahman, T., Bhanushali, A. B., Nadal, L. L., Pierce, G., \& Patel, A. A. (2015, December 3). Weight Loss Following Left Gastric Artery Embolization in a Human Population without Malignancy: A Retrospective Review. Journal of Obesity \& Weight Loss Therapy, 1-4. http://dx.doi.org/10.4172/2165-7904.1000285

Apovian C. M. (2016). Naltrexone/bupropion for the treatment of obesity and obesity with Type 2 diabetes. Future cardiology, 12(2), 129-138. https://doi.org/10.2217/fca.15.79

Arepally, A., Barnett, B. P., Montgomery, E., \& Patel, T. H. (2007). Catheter-directed gastric artery chemical embolization for modulation of systemic ghrelin levels in a porcine model: initial experience. Radiology, 244(1), 138-143. https://doi.org/10.1148/radiol.2441060790

Arepally, A., Barnett, B. P., Patel, T. H., Howland, V., Boston, R. C., Kraitchman, D. L., \& Malayeri, A. A. (2008). Catheter-directed gastric artery chemical embolization suppresses systemic ghrelin levels in porcine model. Radiology, 249(1), 127-133. https://doi.org/10.1148/radiol.2491071232

Aronne, L. J., Wadden, T. A., Peterson, C., Winslow, D., Odeh, S., \& Gadde, K. M. (2013). Evaluation of phentermine and topiramate versus phentermine/topiramate extended-release in obese adults. Obesity (Silver Spring, Md.), 21(11), 2163-2171. https://doi.org/10.1002/oby.20584

Aronne, L. J., Wadden, T., Isoldi, K. K., \& Woodworth, K. A. (2009). When prevention fails: obesity treatment strategies. The American journal of medicine, 122(4 Suppl 1), S24-S32. https://doi.org/10.1016/j.amjmed.2009.01.005

Bai, Z. B., Qin, Y. L., Deng, G., Zhao, G. F., Zhong, B. Y., \& Teng, G. J. (2018). Bariatric Embolization of the Left Gastric Arteries for the Treatment of Obesity: 9-Month Data in 5 Patients. Obesity surgery, 28(4), 907-915. https://doi.org/10.1007/s11695-017-2979-9

Baptista, V., \& Wassef, W. (2013). Bariatric procedures: an update on techniques, outcomes and complications. Current opinion in gastroenterology, 29(6), 684-693. https://doi.org/10.1097/MOG.0b013e3283651af2

Bawudun, D., Xing, Y., Liu, W. Y., Huang, Y. J., Ren, W. X., Ma, M., Xu, X. D., \& Teng, G. J. (2012). Ghrelin suppression and fat loss after left gastric artery embolization in canine model. Cardiovascular and interventional radiology, 35(6), 1460-1466. https://doi.org/10.1007/s00270-012-0362-8 
Bennett, J. M., Mehta, S., \& Rhodes, M. (2007). Surgery for morbid obesity. Postgraduate medical journal, 83(975), 8-15. https://doi.org/10.1136/pgmj.2006.048868

Buchwald, H., Avidor, Y., Braunwald, E., Jensen, M. D., Pories, W., Fahrbach, K., \& Schoelles, K. (2004). Bariatric surgery: a systematic review and metaanalysis. JAMA, 292(14), 1724-1737. https://doi.org/10.1001/jama.292.14.1724

Campos, J., Ramos, A., Szego, T., Zilberstein, B., Feitosa, H., \& Cohen, R. (2016). The Role Of Metabolic Surgery For Patients With Obesity Grade I And Type 2 Diabetes Not Controlled Clinically. Arquivos brasileiros de cirurgia digestiva: ABCD = Brazilian archives of digestive surgery, 29 Suppl 1(Suppl 1), 102-106. https://doi.org/10.1590/0102-6720201600S10025

Cawley, J., \& Meyerhoefer, C. (2012). The medical care costs of obesity: an instrumental variables approach. Journal of health economics, 31(1), 219-230. https://doi.org/10.1016/j.jhealeco.2011.10.003

Christian, J. G., Tsai, A. G., \& Bessesen, D. H. (2010). Interpretando as perdas de peso em estudos de modificação do estilo de vida: usando dados categóricos. Jornal internacional de obesidade (2005), 34 (1), 207-209. https://doi.org/10.1038/ijo.2009.213

Coutinho, A. K., \& Glancey, G. R. (2013). Orlistat, an under-recognised cause of progressive renal impairment. Nephrology, dialysis, transplantation: official publication of the European Dialysis and Transplant Association - European Renal Association, 28 Suppl 4, iv172-iv174. https://doi.org/10.1093/ndt/gft066

Cummings D. E. (2006). Ghrelin and the short- and long-term regulation of appetite and body weight. Physiology \& behavior, 89(1), 71-84. https://doi.org/10.1016/j.physbeh.2006.05.022

Cummings, D. E., \& Overduin, J. (2007). Gastrointestinal regulation of food intake. The Journal of clinical investigation, 117(1), 13-23. https://doi.org/10.1172/JCI30227

Cummings, D. E., Purnell, J. Q., Frayo, R. S., Schmidova, K., Wisse, B. E., \& Weigle, D. S. (2001). A preprandial rise in plasma ghrelin levels suggests a role in meal initiation in humans. Diabetes, 50(8), 1714-1719. https://doi.org/10.2337/diabetes.50.8.1714

Cummings, D. E., Weigle, D. S., Frayo, R. S., Breen, P. A., Ma, M. K., Dellinger, E. P., \& Purnell, J. Q. (2002). Plasma ghrelin levels after diet-induced weight loss or gastric bypass surgery. The New England journal of medicine, 346(21), 1623-1630. https://doi.org/10.1056/NEJMoa012908

Elens, S., Roger, T., Elens, M., Rommens, J., Sarafidis, A., Capelluto, E., \& Delcour, C. (2019). Gastric Embolization as Treatment for Overweight Patients; Efficacy and Safety. Cardiovascular and interventional radiology, 42(4), 513-519. https://doi.org/10.1007/s00270-018-2130-x

English, P. J., Ghatei, M. A., Malik, I. A., Bloom, S. R., \& Wilding, J. P. (2002). Food fails to suppress ghrelin levels in obese humans. The Journal of clinical endocrinology and metabolism, 87(6), 2984. https://doi.org/10.1210/jcem.87.6.8738

Finucane, M. M., Stevens, G. A., Cowan, M. J., Danaei, G., Lin, J. K., Paciorek, C. J., Singh, G. M., Gutierrez, H. R., Lu, Y., Bahalim, A. N., Farzadfar, F., Riley, L. M., Ezzati, M., \& Global Burden of Metabolic Risk Factors of Chronic Diseases Collaborating Group (Body Mass Index) (2011). National, regional, and global trends in body-mass index since 1980: systematic analysis of health examination surveys and epidemiological studies with 960 country-years and 9. 1 million participants. Lancet (London, England), 377(9765), 557-567. https://doi.org/10.1016/S0140-6736(10)62037-5

Flegal, K. M., Carroll, M. D., Ogden, C. L., \& Curtin, L. R. (2010). Prevalence and trends in obesity among US adults, 1999-2008. JAMA, 303(3), 235-241. https://doi.org/10.1001/jama.2009.2014

Fu, Y., \& Kraitchman, D. L. (2020). Rationale and Preclinical Data Supporting Bariatric Arterial Embolization. Techniques in vascular and interventional radiology, 23(1), 100656. https://doi.org/10.1016/j.tvir.2020.100656

Fu, Y., Weiss, C. R., Paudel, K., Shin, E. J., Kedziorek, D., Arepally, A., Anders, R. A., \& Kraitchman, D. L. (2018). Bariatric Arterial Embolization: Effect of Microsphere Size on the Suppression of Fundal Ghrelin Expression and Weight Change in a Swine Model. Radiology, 289(1), 83-89. https://doi.org/10.1148/radiol.2018172874

Gadde, K. M., Allison, D. B., Ryan, D. H., Peterson, C. A., Troupin, B., Schwiers, M. L., \& Day, W. W. (2011). Effects of low-dose, controlled-release, phentermine plus topiramate combination on weight and associated comorbidities in overweight and obese adults (CONQUER): a randomised, placebocontrolled, phase 3 trial. Lancet (London, England), 377(9774), 1341-1352. https://doi.org/10.1016/S0140-6736(11)60205-5

Garcia-Reyes, K., \& Fischman, A. (2020). Anatomical and Technical Considerations for Bariatric Embolization. Techniques in vascular and interventional radiology, 23(1), 100657. https://doi.org/10.1016/j.tvir.2020.100657

Garvey, W. T., Ryan, D. H., Look, M., Gadde, K. M., Allison, D. B., Peterson, C. A., Schwiers, M., Day, W. W., \& Bowden, C. H. (2012). Two-year sustained weight loss and metabolic benefits with controlled-release phentermine/topiramate in obese and overweight adults (SEQUEL): a randomized, placebo-controlled, phase 3 extension study. The American journal of clinical nutrition, 95(2), 297-308. https://doi.org/10.3945/ajcn.111.024927

Glandt, M., \& Raz, I. (2011). Present and future: pharmacologic treatment of obesity. Journal of obesity, 2011, 636181. https://doi.org/10.1155/2011/636181

González-Muniesa, P., Mártinez-González, M. A., Hu, F. B., Després, J. P., Matsuzawa, Y., Loos, R., Moreno, L. A., Bray, G. A., \& Martinez, J. A. (2017). Obesity. Nature reviews. Disease primers, 3, 17034. https://doi.org/10.1038/nrdp.2017.34

Gregorczyk, M., \& Agnieszka, J. (2015, September 3). Arterial supply of gastric fundus in human - final results. MEDtube Science, 30-34. https://medtube.net/science/arterial-supply-of-gastric-fundus-in-human-final-results/

Gunn, A. J., \& Oklu, R. (2014). A preliminary observation of weight loss following left gastric artery embolization in humans. Journal of obesity, 2014, 185349. https://doi.org/10.1155/2014/185349

Gunn, A. J., \& Weiss, C. R. (2019). Is There a Role for Bariatric Embolization in the Treatment of the Diabetic Patient?. Journal of vascular and interventional radiology: JVIR, 30(6), 797-800. https://doi.org/10.1016/j.jvir.2019.03.009 
Hafezi-Nejad, N., Bailey, C. R., \& Weiss, C. R. (2020). Bariatric Embolization: A Narrative Review of Clinical Data From Human Trials. Techniques in vascular and interventional radiology, 23(1), 100658. https://doi.org/10.1016/j.tvir.2020.100658

Hendricks, E. J., Srisurapanont, M., Schmidt, S. L., Haggard, M., Souter, S., Mitchell, C. L., De Marco, D. G., Hendricks, M. J., Istratiy, Y., \& Greenway, F. L. (2014). Addiction potential of phentermine prescribed during long-term treatment of obesity. International journal of obesity (2005), 38(2), 292-298. https://doi.org/10.1038/ijo.2013.74

Hvizdos, K. M., \& Markham, A. (1999). Orlistat: a review of its use in the management of obesity. Drugs, 58(4), 743-760. https://doi.org/10.2165/00003495$199958040-00015$

Jones, L. R., Wilson, C. I., \& Wadden, T. A. (2007). Lifestyle modification in the treatment of obesity: an educational challenge and opportunity. Clinical pharmacology and therapeutics, 81(5), 776-779. https://doi.org/10.1038/sj.clpt.6100155

Kim, D. J., Raman, H. S., Salter, A., Ramaswamy, R., Gunn, A. J., Weiss, C. R., \& Akinwande, O. (2018). Analysis of weight changes after left gastric artery embolization in a cancer-naive population. Diagnostic and interventional radiology (Ankara, Turkey), 24(2), 94-97. https://doi.org/10.5152/dir.2018.17412

Kim, J. M., Kim, M. D., Han, K., Muqmiroh, L., Kim, S. U., Kim, G. M., Kwon, J., Park, S. I., Won, J. Y., \& Lee, D. Y. (2017). Bariatric Arterial Embolization with Non-spherical Polyvinyl Alcohol Particles for Ghrelin Suppression in a Swine Model. Cardiovascular and interventional radiology, 40(5), 744-749. https://doi.org/10.1007/s00270-017-1600-x

Kipshidze, N., Archvadze, A., Bertog, S., Leon, M. B., \& Sievert, H. (2015). Endovascular Bariatrics: First in Humans Study of Gastric Artery Embolization for Weight Loss. JACC. Cardiovascular interventions, 8(12), 1641-1644. https://doi.org/10.1016/j.jcin.2015.07.016

Kojima, M., Hosoda, H., Date, Y., Nakazato, M., Matsuo, H., \& Kangawa, K. (1999). Ghrelin is a growth-hormone-releasing acylated peptide from stomach. Nature, 402(6762), 656-660. https://doi.org/10.1038/45230

Kumar, N., \& Thompson, C. C. (2011). Endoscopic solutions for weight loss. Current opinion in gastroenterology, 27(5), 407-411. https://doi.org/10.1097/MOG.0b013e328349e240

Maahs, D., de Serna, D. G., Kolotkin, R. L., Ralston, S., Sandate, J., Qualls, C., \& Schade, D. S. (2006). Randomized, double-blind, placebo-controlled trial of orlistat for weight loss in adolescents. Endocrine practice: official journal of the American College of Endocrinology and the American Association of Clinical Endocrinologists, 12(1), 18-28. https://doi.org/10.4158/EP.12.1.18

Madoff D. C. (2013). Science to practice: can transarterial embolotherapy be used as a viable alternative to treat obesity?. Radiology, 266(2), 369-371 https://doi.org/10.1148/radiol.12122561

Moher, D., Liberati, A., Tetzlaff, J., Altman, D. G., \& Prisma Group. (2009). Preferred reporting items for systematic reviews and meta-analyses: the PRISMA statement. PLoS med, 6(7), e1000097

O'Neil, P. M., Smith, S. R., Weissman, N. J., Fidler, M. C., Sanchez, M., Zhang, J., Raether, B., Anderson, C. M., \& Shanahan, W. R. (2012). Randomized placebo-controlled clinical trial of lorcaserin for weight loss in type 2 diabetes mellitus: the BLOOM-DM study. Obesity (Silver Spring, Md.), 20(7), 14261436. https://doi.org/10.1038/oby.2012.66

Park, Y. S., Ahn, S. H., Suh, Y. S., \& Kim, H. H. (2020). Less Invasive Bariatric/Metabolic Procedures for Weight Reduction and Glucose Control. Journal of obesity \& metabolic syndrome, 29(2), 110-113. https://doi.org/10.7570/jomes20019

Paxton, B. E., Kim, C. Y., Alley, C. L., Crow, J. H., Balmadrid, B., Keith, C. G., Kankotia, R. J., Stinnett, S., \& Arepally, A. (2013). Bariatric embolization for suppression of the hunger hormone ghrelin in a porcine model. Radiology, 266(2), 471-479. https://doi.org/10.1148/radiol.12120242

Piché, M. È., Auclair, A., Harvey, J., Marceau, S., \& Poirier, P. (2015). How to choose and use bariatric surgery in 2015. The Canadian journal of cardiology, 31(2), 153-166. https://doi.org/10.1016/j.cjca.2014.12.014

Pirlet, C., Ruzsa, Z., Costerousse, O., Nemes, B., Merkely, B., Poirier, P., \& Bertrand, O. F. (2019). Transradial left gastric artery embolization to treat severe obesity: A pilot study. Catheterization and cardiovascular interventions : official journal of the Society for Cardiac Angiography \& Interventions, 93(3), 365370. https://doi.org/10.1002/ccd.27846

Pi-Sunyer, X., Astrup, A., Fujioka, K., Greenway, F., Halpern, A., Krempf, M., Lau, D. C., le Roux, C. W., Violante Ortiz, R., Jensen, C. B., Wilding, J. P., \& SCALE Obesity and Prediabetes NN8022-1839 Study Group (2015). A Randomized, Controlled Trial of $3.0 \mathrm{mg}$ of Liraglutide in Weight Management. The New England journal of medicine, 373(1), 11-22. https://doi.org/10.1056/NEJMoa1411892

Prochaska, J. M., Flye, M. W., \& Johnsrude, I. S. (1973). Left Gastric Artery Embolization for Control of Gastric Bleeding: A Complication. Radioogy Journal, 107(3), 521-522. https://doi.org/10.1148/107.3.521

Reoch, J., Mottillo, S., Shimony, A., Filion, K. B., Christou, N. V., Joseph, L., Poirier, P., \& Eisenberg, M. J. (2011). Safety of laparoscopic vs open bariatric surgery: a systematic review and meta-analysis. Archives of surgery (Chicago, Ill.: 1960), 146(11), 1314-1322. https://doi.org/10.1001/archsurg.2011.270

Salsamendi, J., Pereira, K., Kang, K., \& Fan, J. (2015). Minimally invasive percutaneous endovascular therapies in the management of complications of nonalcoholic fatty liver disease (NAFLD): A case report. Journal of radiology case reports, 9(9), 36-43. https://doi.org/10.3941/jrcr.v9i9.2557

Stefater, M. A., Kohli, R., \& Inge, T. H. (2013). Advances in the surgical treatment of morbid obesity. Molecular aspects of medicine, 34(1), 84-94. https://doi.org/10.1016/j.mam.2012.10.006

Syed, M. I., Morar, K., Shaikh, A., Craig, P., Khan, O., Patel, S., \& Khabiri, H. (2016). Gastric Artery Embolization Trial for the Lessening of Appetite Nonsurgically (GET LEAN): Six-Month Preliminary Data. Journal of vascular and interventional radiology: JVIR, 27(10), 1502-1508. https://doi.org/10.1016/j.jvir.2016.07.010 
Takahashi, E. A., Takahashi, N., Reisenauer, C. J., Moynagh, M. R., \& Misra, S. (2019). Body composition changes after left gastric artery embolization in overweight and obese individuals. Abdominal radiology (New York), 44(7), 2627-2631. https://doi.org/10.1007/s00261-019-02002-6

Takiguchi, S., Adachi, S., Yamamoto, K., Morii, E., Miyata, H., Nakajima, K., Yamasaki, M., Kangawa, K., Mori, M., \& Doki, Y. (2012). Mapping analysis of ghrelin producing cells in the human stomach associated with chronic gastritis and early cancers. Digestive diseases and sciences, 57(5), $1238-1246$. https://doi.org/10.1007/s10620-011-1986-2

Torgerson, J. S., Hauptman, J., Boldrin, M. N., \& Sjöström, L. (2004). XENical in the prevention of diabetes in obese subjects (XENDOS) study: a randomized study of orlistat as an adjunct to lifestyle changes for the prevention of type 2 diabetes in obese patients. Diabetes care, 27(1), 155-161. https://doi.org/10.2337/diacare.27.1.155

Vaidya, S., Tozer, K. R., \& Chen, J. (2008). An overview of embolic agents. Seminars in interventional radiology, 25(3), 204-215. https://doi.org/10.1055/s$0028-1085930$

Vairavamurthy, J., Cheskin, L. J., Kraitchman, D. L., Arepally, A., \& Weiss, C. R. (2017). Current and cutting-edge interventions for the treatment of obese patients. European journal of radiology, 93, 134-142. https://doi.org/10.1016/j.ejrad.2017.05.019

Verdam, F. J., Schouten, R., Greve, J. W., Koek, G. H., \& Bouvy, N. D. (2012). An update on less invasive and endoscopic techniques mimicking the effect of bariatric surgery. Journal of obesity, 2012, 597871. https://doi.org/10.1155/2012/597871

Weir, M. A., Beyea, M. M., Gomes, T., Juurlink, D. N., Mamdani, M., Blake, P. G., Wald, R., \& Garg, A. X. (2011). Orlistat and acute kidney injury: an analysis of 953 patients. Archives of internal medicine, 171(7), 703-704. https://doi.org/10.1001/archinternmed.2011.103

Weiss, C. R., \& Kathait, A. S. (2017). Bariatric embolization: a new and effective option for the obese patient? Expert review of gastroenterology \& hepatology, 11(4), 293-302. https://doi.org/10.1080/17474124.2017.1294060

Weiss, C. R., Akinwande, O., Paudel, K., Cheskin, L. J., Holly, B., Hong, K., Fischman, A. M., Patel, R. S., Shin, E. J., Steele, K. E., Moran, T. H., Kaiser, K., Park, A., Shade, D. M., Kraitchman, D. L., \& Arepally, A. (2017). Clinical Safety of Bariatric Arterial Embolization: Preliminary Results of the BEAT Obesity Trial. Radiology, 283(2), 598-608. https://doi.org/10.1148/radiol.2016160914

Weiss, C. R., Gunn, A. J., Kim, C. Y., Paxton, B. E., Kraitchman, D. L., \& Arepally, A. (2015). Bariatric embolization of the gastric arteries for the treatment of obesity. Journal of vascular and interventional radiology: JVIR, 26(5), 613-624. https://doi.org/10.1016/j.jvir.2015.01.017

Williams, E. P., Mesidor, M., Winters, K., Dubbert, P. M., \& Wyatt, S. B. (2015). Overweight and Obesity: Prevalence, Consequences, and Causes of a Growing Public Health Problem. Current obesity reports, 4(3), 363-370. https://doi.org/10.1007/s13679-015-0169-4

World Health Organization, W. H. O. (2000). Obesity: preventing and managing the global epidemic (WHO Technical Report Series 894). Report of a WHO Consultation.

World Health Organization, W. H. O. (2016). Obesity and overweight. World Health Organization website. Retrieved March 24, 2020, from http://www.who.int/mediacentre/factsheets/fs311/en/

Wren, A. M., \& Bloom, S. R. (2007). Gut hormones and appetite control. Gastroenterology, 132(6), 2116-2130. https://doi.org/10.1053/j.gastro.2007.03.048

Wren, A. M., Seal, L. J., Cohen, M. A., Brynes, A. E., Frost, G. S., Murphy, K. G., Dhillo, W. S., Ghatei, M. A., \& Bloom, S. R. (2001). Ghrelin enhances appetite and increases food intake in humans. The Journal of clinical endocrinology and metabolism, 86(12), 5992. https://doi.org/10.1210/jcem.86.12.8111

Yanovski, S. Z., \& Yanovski, J. A. (2014). Long-term drug treatment for obesity: a systematic and clinical review. JAMA, 311(1), 74-86. https://doi.org/10.1001/jama.2013.281361

Zaitoun, M., Basha, M., Hassan, F., Elsayed, S. B., Farag, A. A., Amer, M., Aly, S. A., \& Zaitoun, N. (2019). Left Gastric Artery Embolization in Obese, Prediabetic Patients: A Pilot Study. Journal of vascular and interventional radiology: JVIR, 30(6), 790-796. https://doi.org/10.1016/j.jvir.2019.02.010

Zhong, B. Y., Abiola, G., \& Weiss, C. R. (2018). Bariatric Arterial Embolization for Obesity: A Review of Early Clinical Evidence. Cardiovascular and interventional radiology, 41(11), 1639-1647. https://doi.org/10.1007/s00270-018-1996-y

Zhong, B. Y., Li, P. C., \& Ni, C. F. (2019). Research progress of bariatric embolization for treatment of obesity. Chinese medical journal, 132(7), 880-882. https://doi.org/10.1097/CM9.00000000000001552 